\title{
Isolationism is not the answer to bioterrorism
}

\author{
Increased support for research in the developing world would be a better strategy.
}

Sir - It has been suggested that the best way for the United States to keep biotechnology-enhanced biological weapons away from terrorist groups is for it to support related research and training only for US scientists (see, for example, Nature 414, 3-4; 2001). We do not believe such an isolationist attitude to be useful. Increasing research support and training in biotechnology and genomics to scientists in the developing world may be the best way to prevent bioweapon attacks on the United States and its allies.

Inadequate funding by northern scientific bodies - of other scientific disciplines as well as genomics and biotechnology - marginalizes southern scientists and widens the gap between northern and southern scientists' mindsets. If southern scientists are given a stake in the northern system, through sponsored research opportunities, its scientists will be less likely to want to help terrorist actions against northern interests.

Southern scientists are, of course, making significant novel contributions to science, which could include assisting the development of biodefence strategies. Their contributions are not only welcome, but enhanced northern research support could also challenge the stereotype that the north does not have the interests of the south at heart - a view propagated by anti-northern extremist groups. Scientists everywhere, south or north, need to be aware of the regulatory and ethical implications of bioweapon proliferation. Sponsored training by northern funding agencies is the best way to achieve this end.

Such training in genomics and biotechnology could be made conditional upon such scientists passing stringent intelligence review and verification by the sponsoring institution or its authorized representative, and their states being signatories to chemical- and biologicalwarfare conventions and protocols. Given the potential trade and investment opportunities that come with a skilled, biotechnologically competent workforce, sponsoring advanced training of southern scientists in genomics or biotechnology could serve as an incentive for countries to sign and comply with the conventions.

The Fogarty International Center of the US National Institutes of Health (NIH) has budgeted approximately $\$ 45$ million for the training of international scientists for fiscal year 2002-03. Although this approach is commendable, apart from the dilution of the money over all the subdisciplines in health, the amount is minuscule compared with the $\$ 1.75$ billion the US government has allocated to the NIH for biodefence research alone in 2003. Increasing research support and training in biotechnology and genomics would make it easier for northern scientists to say to their southern colleagues: "We're acting with you, so don't act against us."

Jerome A. Singh ${ }^{\star} \dagger$, Peter A. Singer $\dagger$

${ }^{\star}$ Howard College School of Law, University of

Natal, Durban, 4041, South Africa

$\dagger$ University of Toronto Joint Centre for Bioethics, 88 College Street, Toronto M5G 1L4, Canada

\section{Schools can be inspired by a summer of science}

Sir-We appreciated reading your recommendation that other universities should promote initiatives like those offered in the United States, to "give young people enough of a glimpse of the world of science to be enticed further into it". In particular, we liked your suggestion that research facilities should invite secondaryschool students to their laboratories during the summer holidays (see Nature 419, 233; 2002).

In Portugal, research institutes have been providing summer internships for secondary-school students for several years now, with the support of European Regional Development Funds and the Portuguese government (see www. cienciaviva.pt). Students work in research laboratories, where they are given specific simple scientific tasks, sometimes including field work, for one week or more. The aim is to give them a clear idea of the realities of research in topics including mathematics, biotechnology, robotics and cancer. Several of the best Portuguese scientific institutes participate in this activity on a regular basis, and both students and researchers have found it to be a very positive experience.

Last summer, a similar initiative was extended to secondary-school teachers, who are given a chance to work with state- of-the-art laboratory equipment and learn about the most recent developments in scientific and technological research. Rosalia Vargas, Ana Noronha Ciência Viva - National Agency for Scientific and Technological Culture, Pavilion of Knowledge, Parque das Nações, Alameda dos Oceanos, 1990-223 Lisboa, Portugal

\section{What has posterity done for us? It's not the point}

Sir - To understand why many physical scientists regard economists with scepticism, one need look no further than the Concepts essay on discounting ("An eye on the future" Nature 419, 673-674; 2002) by L. H. Goulder and R. N. Stavins. After describing an example in which a \$4-billion investment now would prevent us from causing $\$ 800$ billion of environmental damage 100 years hence, they ask: "If future generations do not actually compensate the present one, is it still appropriate to enact the policy?"

Who but an economist could imagine that future generations would owe us an impossible debt for not damaging their environment? Isn't it we who owe future generations a sound environment? Discounting provides a well-defined measure relating present and future sums.
The problem is that this measure is not particularly useful for problems involving intergenerational transfer.

\section{Ken Caldeira}

Climate and Carbon Cycle Group, Energy and Environment Directorate, Lawrence Livermore National Laboratory, 7000 East Avenue, L-103, Livermore, California 94550, USA

Goulder and Stavins reply - We agree with Caldeira's view that it would be unfair to require future generations to pay the present generation for the costs of current climate policy. Our example was meant to illustrate complications that arise in evaluating policy options involving winners and losers.

Caldeira goes on to claim that discounting (and benefit-cost analysis) is not useful for problems with intergenerational impacts. Here we differ. The aggregate policy-generated gains and losses, translated by discounting into comparable units, are highly relevant for assessing public policies. But we emphasize here, as in our essay, that other considerations - including attention to other criteria of fairness - are important in policy evaluation.

Lawrence H. Goulder*, Robert N. Stavins $\dagger$

*Department of Economics, Stanford University, Stanford, California 94305-6072

$\dagger$ John F. Kennedy School of Government, Harvard University, Cambridge, Massachusetts 02138, USA 